\title{
Hitting a Boundary Point by Diffusions in the Closed Half Space*
}

\author{
S. RAMASUBRAMANIAN \\ Indian Statistical Institute, Bangalore, India and \\ Center for Stochastic Processes, University of North Carolina \\ Communicated by G. Kallianpur
}

\begin{abstract}
It is proved that a nondegenerate diffusion process in the closed half space $\bar{G}=\left\{x \in R^{d}: x_{1} \geqslant 0\right\}$, where $d \geqslant 2$, with Wentzell's boundary conditions does not hit any specified point on the boundary. 1986 Academic Press, Inc.
\end{abstract}

It is known that a Brownian motion in the unit sphere, with normal reflection at the boundary, does not hit a specified point on the boundary (see McKean [4]). The aim of this article is to prove that a non-degenerate diffusion in the closed half spacc, with certain Wentzell-type boundary conditions, does not hit a point on the boundary specified in advance. We also give an application to a boundary value problem.

Let $G=\left\{x=\left(x_{1}, \ldots, x_{d}\right): x_{1}>0\right\}, \partial G=\left\{x \in \mathbb{R}^{d}: x_{1}=0\right\}$ and $\bar{G}=G \cup \partial G$, where $d \geqslant 2$. We have the coefficients $a, b$ defined on $\bar{G}$, and $\alpha, \gamma, \rho$ defined on $\partial G$, satisfying one of the following two sets of conditions.

Conditions I. (I 1) For each $x \in \bar{G}, a(x)=\left(\left(a_{i j}(x)\right)_{1 \leqslant i, j \leqslant d}\right.$ is a $d \times d$ real symmetric positive definite matrix; $a(\cdot)$ is bounded and continuous; $a^{-1}(\cdot)$ is also bounded and continuous.

(I 2) $b(\cdot)=\left(b_{1}(\cdot), \ldots, b_{d}(\cdot)\right)$ is a bounded and continuous $\mathbb{R}^{d}$-valued function on $\bar{G}$.

(I 3) $\gamma(\cdot)=\left(\gamma_{2}(\cdot), \ldots, \gamma_{d}(\cdot)\right)$ is an $\mathbb{R}^{d-1}$-valued function on $\partial G$; $\gamma_{j} \in C_{b}^{2}(\partial G)$ for $j=2, \ldots, d$.

(I 4) $\alpha \equiv 0$ as a $(d-1) \times(d-1)$ matrix.

(I 5) $\rho \equiv 0$; or $\rho$ is a bounded locally Lipschitz function which is strictly positive at each point of $\partial G$.

* This research partially supported by the Air Force Office of Scientific Research Contract F49620 82 C 0009.

Received February 1, 1984; revised July 25, 1985

AMS 1980 subject classification: Primary $60 \mathrm{~J} 60$. 
The set of alternative conditions is

Conditions II. (II 1) In addition to (I 1) we assume that for each $x \in \bar{G}$, there exists a $d \times d$ real symmetric positive definite matrix $\sigma(x)=\left(\left(\sigma_{i j}(x)\right)\right)_{1 \leqslant i, j \leqslant d}$ such that $a(x)=\sigma(x) \sigma^{*}(x), \sigma(\cdot)$ is bounded and continuous, and $\sigma^{-1}(\cdot)$ is also bounded and continuous.

(II 2) Same as (I 2).

(II 3) $\gamma(\cdot)=\left(\gamma_{2}(\cdot), \ldots, \gamma_{d}(\cdot)\right)$ is an $\mathbb{R}^{d-1}$-valued bounded and continuous function on $\partial G$.

(II 4) For each $x \in \partial G, \alpha(x)=\left(\left(\alpha_{i j}(x)\right)\right)_{2 \leqslant i, j \leqslant d}$ is a $(d-1) \times(d-1)$ real symmetric positive definite matrix, and there exists a $(d-1) \times(d-1)$ real symmetric positive definite matrix $\tilde{\sigma}(x)=\left(\left(\tilde{\sigma}_{i j}(x)\right)\right)_{2 \leqslant i, j \leqslant d}$ such that $\alpha(x)=\tilde{\sigma}(x) \cdot \tilde{\sigma}^{*}(x) . \tilde{\sigma}(\cdot)$ and $\tilde{\sigma}^{-1}(\cdot)$ are bounded and continuous.

(II 5) Same as (I 5).

Define

$$
L=\frac{1}{2} \sum_{i, j=1}^{d} a_{i j}(x) \frac{\partial^{2}}{\partial x_{i} \partial x_{i}}+\sum_{i=1}^{d} b_{i}(x) \frac{\partial}{\partial x_{i}}
$$

and

$$
J=\frac{\partial}{\partial x_{1}}+\frac{1}{2} \sum_{i, j=2}^{d} \alpha_{i j}(x) \frac{\partial^{2}}{\partial x_{i} \partial x_{j}}+\sum_{i=2}^{d} \gamma_{i}(x) \frac{\partial}{\partial x_{i}} .
$$

Let $\Omega=C([0, \infty): \bar{G})$ be endowed with the topology of uniform convergence on compacta and the natural Borel structure.

Under conditions less restrictive than the set of Conditions I, Stroock and Varadhan [7], have established the existence of a unique solution to the submartingale problem corresponding to the coefficients $a, b, \gamma, \rho$. Following Watanabe [9], Nakao and Shiga [6] have established the existence of a unique solution to the stochastic differential equation corresponding to the coefficients $a, b, \alpha, \gamma, \rho$ under conditions less restrictive than the set of Conditions II. The equivalences of these two formulations can be found in El Karoui [3]. (Here uniqueness is in the sense of law.)

So, when Conditions I or II hold, for each $x \in \bar{G}$ there exists a unique probability measure $P_{x}$ on $\Omega$ such that

(1) $P_{x}\{X(t) \in \bar{G}$ for all $t \geqslant 0$ and $X(0)=x\}=1$,

(2) $f(X(t))-\int_{0}^{t}\left[I_{G} \cdot(L f)\right](X(u)) d u$

is a $P_{x}$-submartingale for any $f \in C_{b}^{2}\left(\mathbb{R}^{d}\right)$ satisfying $J f \geqslant 0$ on $\hat{\partial} G$, and where $X(t)$ denotes the $t$ th coordinate map on $\Omega$; also the process $X(t)$ is strong 
Markov and Feller continuous. Further, there exists a continuous, nondecreasing, non-anticipating process $\xi(t)$ on $\Omega$ such that

$$
\text { (i) } \xi(t)=\int_{0}^{t} I_{\partial G}(X(u)) d \xi(u)
$$

and

(ii) $f(X(t))-\int_{0}^{t}\left[I_{G} \cdot(L f)\right](X(u)) d u-\int_{0}^{t} J f(X(u)) d \xi(u)$

is a $P_{x}$-martingale for every $f \in C_{b}^{2}\left(\mathbb{R}^{d}\right)$. We shall call the family $\left\{P_{x}: x \in \bar{G}\right\}$ the diffusion corresponding to $(L, J)$.

We first prove a theorem which effectively reduces the problem to the case of normal reflection; this theorem may be of independent interest. But, we first need a few lemmas.

Lemma 1. Let $g: \mathbb{R}^{m} \rightarrow \mathbb{R}^{m}$ be a bounded and continuous function (i.e., the image of $g$ is contained in a compact set). Define $g_{1}: \mathbb{R}^{m} \rightarrow \mathbb{R}^{m}$ by $g_{1}(x)=x+g(x)$. Then $g_{1}$ is onto.

Proof. Let $z \in \mathbb{R}^{m}$ be fixed. Define $h_{z}: \mathbb{R}^{m} \rightarrow \mathbb{R}^{m}$ by $h_{z}(x)=-g(x)+z$. Since the range of $h_{z}$ is contained in a compact set, by Brouwers fixed point theorem, there exists $x \in \mathbb{R}^{m}$ such that $h_{z}(x)=x$, i.e., $z=x+g(x)$. This shows that $g_{1}$ is onto.

Lemma 2. Let Conditions I hold. There exists a $C^{2}$-diffeomorphism $T: \bar{G} \rightarrow \bar{G}$, given by $\left(y_{1}, y_{2}, \ldots, y_{d}\right)=T\left(z_{1}, z_{2}, \ldots, z_{d}\right)$, such that the following hold:

(i) $T$ is identity on $\partial G$.

(ii) Under $T^{-1}, \quad J=\partial / \partial y_{1}+\sum_{i=2}^{d} \gamma_{i}(y) \partial / \partial y_{i}$ is transformed to $\tilde{J}=\partial / \partial z_{1}$ on $\partial G$.

(iii) $L$, given by (1) (in the variables $y_{1}, \ldots, y_{d}$ ), is transformed to a strictly elliptic operator $\tilde{L}$ with bounded coefficients (in the variables $\left.z_{1}, z_{2}, \ldots, z_{d}\right)$ under $T^{-1}$; and $\tilde{L}$ has a representation like (1).

Proof. By condition (I 1) there exist constants $a_{0}>0, M>0$ such that

$$
\left|a_{i j}(x)\right| \leqslant M \quad \text { for all } x \in \bar{G}, 1 \leqslant i, j \leqslant d,
$$

and

$$
a_{0}=\inf \{\text { eigenvalues of } a(x): x \in \bar{G}\} \text {. }
$$


We first consider the case when there is a constant $\mu$ such that

$$
\left|\gamma_{j}\right|,\left|\frac{\partial \gamma_{j}}{\partial x_{k}}\right|,\left|\frac{\partial^{2} \gamma_{j}}{\partial x_{k} \partial_{x_{i}}}\right| \leqslant \mu<\frac{a_{0}}{8 M d^{3}} .
$$

For $x=\left(x_{1}, x_{2}, \ldots, x_{d}\right)$, let $\tilde{x}=\left(0, x_{2}, \ldots, x_{d}\right)$. Note that by (4), for $\tilde{x}, \tilde{x}^{\prime} \in \partial G$, we have

$$
\left|\gamma(\tilde{x})-\gamma\left(\tilde{x}^{\prime}\right)\right|^{2} \leqslant(d-1) \mu^{2}\left|\tilde{x}-\tilde{x}^{\prime}\right|^{2} .
$$

Let $\lambda$ be such that $0<\lambda<1 /(2 d \mu)$; let $\phi$ be a smooth function on $(-1, \infty)$ such that $\phi$ is non-decreasing, $\left|\phi^{\prime}\right| \leqslant 1$, and $\phi(v)=v$ if $v \leqslant \frac{1}{2} \lambda$, and $\phi(v)=\lambda$ if $v \geqslant \lambda$. Define $T: \bar{G} \rightarrow \bar{G}$ by

$$
\begin{aligned}
\left(y_{1}, y_{2}, \ldots, y_{d}\right) & =T\left(z_{1}, z_{2}, \ldots, z_{d}\right) \\
& =\left(z_{1}, z_{2}, \ldots, z_{d}\right)+\phi\left(z_{1}\right)\left(0, \gamma_{2}(\tilde{z}), \ldots, \gamma_{d}(\tilde{z})\right) .
\end{aligned}
$$

We claim that $T$ is one-to-one; indeed, let $T\left(z_{1}, z_{2}, \ldots, z_{d}\right)=$ $T\left(z_{1}^{\prime}, z_{2}^{\prime}, \ldots, z_{d}^{\prime}\right)$. By $(6)$, it is clear that $z_{1}=z_{1}^{\prime}$; and hence, $\phi\left(z_{1}\right)=\phi\left(z_{1}^{\prime}\right)$. Therefore $\tilde{z}+\phi\left(z_{1}\right) \gamma(\tilde{z})=\tilde{z}^{\prime}+\phi\left(z_{1}\right) \gamma\left(\tilde{z}^{\prime}\right)$. Consequently by (5),

$$
\left|\tilde{z}-\tilde{z}^{\prime}\right|=\phi\left(z_{1}\right)\left|\gamma(\tilde{z})-\gamma\left(\tilde{z}^{\prime}\right)\right| \leqslant \hat{\lambda} \sqrt{d-1} \mu\left|\tilde{z}-\tilde{z}^{\prime}\right|<\left|\tilde{z}-\tilde{z}^{\prime}\right|,
$$

which is a contradiction unless $\tilde{z}=\tilde{z}^{\prime}$. Thus $T$ is $1-1$. By Lemma $1, T$ is onto. (Actually $T$ is one-one and onto on every $\left\{z_{1}=\right.$ constant $\}$.) Since $\phi(0)=0$, it follows that $T$ is identity on $\partial G$.

Since $T$ is a bijection, from (6), we may write

$$
\begin{aligned}
\left(z_{1}, z_{2}, \ldots, z_{d}\right) & \left.=y_{1}, y_{2}, \ldots, y_{d}\right)-\phi\left(z_{1}\right)\left(0, \gamma_{2}(\tilde{z}), \ldots, \gamma_{d}(\tilde{z})\right) \\
& =\left(y_{1}, y_{2}, \ldots, y_{d}\right)-\phi\left(y_{1}\right)\left(0, \theta_{2}(y), \ldots, \theta_{d}(y)\right),
\end{aligned}
$$

where $\theta_{j}(y)=\gamma_{j}(\tilde{z}(y))$, with $\tilde{z}$ expressed as a function of $y$.

Since $\gamma_{j}$ 's are twice continuously differentiable, by inverse function theorem it follows that the transformation $T$ is a $C^{2}$-diffeomorphism and its inverse is also a $C^{2}$-diffeomorphism. Thus, $\theta_{j}$ 's are twice differentiable as functions of $y$.

Next, we claim that

$$
\left|\frac{\partial \theta_{j}}{\partial y_{p}}\right| \leqslant \frac{\sqrt{d} \mu}{1-(d \lambda \mu)} .
$$

To that end, set $\gamma_{1} \equiv 0, \quad \theta_{1} \equiv 0 ; \quad \partial_{p} \theta=\left(\partial \theta_{1} / \partial y_{p}, \ldots, \partial \theta_{d} / \partial y_{p}\right), \quad z_{p} \gamma=$ $\left(\partial \gamma_{1} / \partial z_{p}, \ldots, \partial \gamma_{d} / \partial z_{p}\right)$, for $p=1,2, \ldots, d$. Here it may be noted that $\gamma_{2}, \ldots, \gamma_{d}$ can be considered functions on $\bar{G}$ by making $\left.\gamma_{j}(x)=\gamma_{j}(\tilde{x})\right)$. Let $D_{z} \gamma$ denote the 
$(d \times d)$ matrix given by $\left(D_{z} \gamma\right)_{j k}=\left(\partial \gamma_{j} / \partial z_{k}\right)$. Then a simple computation shows that

$$
\left[I+\phi\left(y_{1}\right)\left(D_{z} \gamma\right)\right] \partial_{p} \theta=\tilde{\partial}_{p} \gamma
$$

where $I$ is the $(d \times d)$ identity matrix. Since $\left|\phi\left(y_{1}\right)\left(\partial \gamma_{j} / \partial z_{k}\right)\right| \leqslant \lambda \mu$ and $d \lambda \mu<\frac{1}{2}$, it follows that $\left[I+\phi\left(y_{1}\right) D_{z} \gamma\right]^{-1}$ exists and

$$
\left\|\left[I+\phi\left(y_{1}\right) D_{z} \gamma\right]^{-1}\right\| \leqslant \frac{1}{(1-(d \lambda \mu))} .
$$

Hence by (4) and (9) we get

$$
\left|\partial_{p} \theta\right| \leqslant \frac{\sqrt{d} \mu}{1-(d \lambda \mu)}
$$

whence (8) follows.

Now for any smooth function $g$, by (7), we obtain

$$
\begin{aligned}
& \frac{\partial g}{\partial y_{1}}=\frac{\partial g}{\partial z_{1}}+\sum_{j=2}^{d}\left[-\phi^{\prime}\left(y_{1}\right) \theta_{j}(y)-\phi\left(y_{1}\right) \frac{\partial \theta_{j}}{\partial y_{1}}\right] \frac{\partial g}{\partial z_{j}}, \\
& \frac{\partial g}{\partial y_{i}}=\left[1-\phi\left(y_{1}\right) \frac{\partial \theta_{i}}{\partial y_{i}}\right] \frac{\partial g}{\partial z_{i}}+\sum_{j \neq 1, i}\left[-\phi\left(y_{1}\right) \frac{\partial \theta_{j}}{\partial y_{i}}\right] \frac{\partial g}{\partial z_{j}},
\end{aligned}
$$

for $i=2, \ldots, d$. Since $\phi(0)=0, \phi^{\prime}(0)=1$, and $\theta_{j}(y)=\gamma_{j}(\tilde{y})$ on $\left\{y_{1}=0\right\}$, it follows from (10) that

$$
\left.\left[\frac{\partial}{\partial y_{1}}+\sum_{i=2}^{d} \gamma_{i}(\tilde{y}) \frac{\partial}{\partial y_{1}}\right]\right|_{y_{1}=0}=\left.\frac{\partial}{\partial z_{1}}\right|_{z_{1}=0} .
$$

This establishes conclusion (ii) of the lemma.

Differentiating again, it can be shown that, for $i, j=1,2, \ldots, d$,

$$
\frac{\partial^{2} g(\cdot)}{\partial y_{i} \partial y_{j}}=\frac{\partial^{2} g(\cdot)}{\partial z_{i} \partial z_{j}}+\sum_{k, l=1}^{d} \delta_{k l}^{i j}(\cdot) \frac{\partial^{2} g(\cdot)}{\partial z_{k} \partial z_{l}}+\text { first-order terms, }
$$

where $\delta_{k l}^{i j}=\delta_{l k}^{\prime l}$; since $|\phi| \leqslant \lambda,\left|\phi^{\prime}\right| \leqslant 1,\left|\theta_{j}\right| \leqslant \mu<1$, by (4), (8), and the calculations leading to (12), it can be proved that

$$
\left|\delta_{k l}^{i j}\right| \leqslant \frac{4}{1-(d \lambda \mu)} \mu .
$$


Now from (12) we get

$$
\begin{aligned}
& \sum_{i, j=1}^{d} a_{i j}(\cdot) \frac{\partial^{2} g(\cdot)}{\partial y_{i} \partial y_{j}}+\sum_{i=1}^{d} b_{i}(\cdot) \frac{\partial g(\cdot)}{\partial y_{i}} \\
& =\sum_{i, j=1}^{d}\left[a_{i j}(\cdot)+\eta_{i j}(\cdot)\right] \frac{\partial^{2} g(\cdot)}{\partial z_{i} \partial z_{j}}+\text { first-order terms }
\end{aligned}
$$

where $\eta_{y j}(\cdot)=\sum_{k, l=1}^{d} a_{i j}(\cdot) \delta_{k l}^{i j}(\cdot)$. In view of (13) it is easily seen that for any $\zeta=\left(\zeta_{1}, \ldots, \zeta_{d}\right)$ in $\mathbb{R}^{d}$,

$$
\left|\sum_{i, j=1}^{d} \eta_{i j} \zeta_{i} \zeta_{j}\right| \leqslant \frac{4 M d^{3}}{1-(d \lambda \mu)} \mu|\zeta|^{2} .
$$

Since $d \lambda \mu<\frac{1}{2}$, we have from (4) and (15),

$$
\sum_{i, j=1}^{d}\left(a_{i j}+\eta_{i j}\right) \zeta_{i} \zeta_{j} \geqslant\left[a_{0}-\frac{4 M d^{3}}{1-(d \hat{\lambda} \mu)} \mu\right]|\zeta|^{2}>0
$$

for any $\zeta \neq 0$. It may be noted that there are no terms of the form $q(\cdot) g(\cdot)$ in (12), and hence in (14). Thus $\widetilde{L}$, given by the right-side of (14), is uniformly elliptic (in the variables $z_{1}, z_{2}, \ldots, z_{d}$ ). This completes the proof in the special case.

In the general case, since $\gamma_{i} \in C_{b}^{2}(\partial G)$, there exists a constant $K_{1}$ such that

$$
\left|\gamma_{i}\right|,\left|\frac{\partial \gamma_{j}}{\partial z_{k}}\right|,\left|\frac{\partial^{2} \gamma_{j}}{\partial z_{k} \partial z_{l}}\right| \leqslant K_{1} .
$$

Choose $K$ large enough that $K_{1} / K<a_{0} /\left(8 M d^{3}\right)$. Note that the diffusion corresponding to $(L, J)$ is also the diffusion corresponding to $(L,(1 / K) J)$.

Set $\hat{z}_{1}=K z_{1}, \hat{z}_{j}=z_{j}, j=2, \ldots, d ; \hat{\gamma}_{j}(\hat{z})=(1 / K) \gamma_{j}(z)$ for $z \in \partial G$. It is then easily seen that the general case is reduced to the previous case with the new ellipticity constant $a_{0}(K \wedge 1)$; also $(1 / K) J$ in the $z$-coordinates is transformed to $\partial / \partial \hat{z}_{1}+\sum_{i=2}^{d} \hat{\gamma}_{j}(\hat{z})\left(\partial / \partial \hat{z}_{j}\right)$ in the $\hat{z}$-coordinates. The lemma now follows in the general case from the special case considered previously.

We can now state our first theorem.

Theorem 1. Let Conditions I hold; let $L, \tilde{L}, J, \tilde{J}, T$ be as in Lemma 2. Let $\left\{P_{y}: y \in \bar{G}\right\}$ be the diffusion corresponding to $(L, J)$. Let $\hat{T}: \Omega \rightarrow \Omega$ be defined by $(\hat{T} w)(t)=T(w(t))$. $\hat{T}$ is a homeomorphism on $\Omega$. Set $\widetilde{P}_{z}=P_{y} \hat{T}$, where $y=T(z)$. Then $\left\{\widetilde{P}_{z}: z \in \bar{G}\right\}$ is the diffusion corresponding to $(\tilde{L}, \tilde{J})$.

Proof. Let $\mathscr{B}_{t}=\sigma\{X(s): 0 \leqslant s \leqslant t\}$ be the natural filtration in $\Omega$. If 
$E \in \mathscr{B}_{t}$, note that $\hat{T} E, \hat{T}^{-1} E \in \mathscr{B}_{t}$. Let $f \in C_{b}^{2}\left(\mathbb{P}^{d}\right)$ be such that $\tilde{J} \geqslant 0$ on $\partial G$. Define $\bar{f}$ by setting $\bar{f}(y)=f\left(T^{-1} y\right)$. Note that $J \bar{f}(y)=\widetilde{J} f(z)$, where $y=T(z)$; consequently $J \bar{f} \geqslant 0$ on $\partial G$. Hence

$$
\bar{f}(X(t))-\int_{0}^{t}\left[I_{G} \cdot(L \bar{f})\right](X(u)) d u
$$

is a $P_{y}$-submartingale (with respect to $\mathscr{B}_{t}$ ).

Note that by Lemma 2

$$
\left[I_{G} \cdot(\tilde{L} f)\right]\left(\hat{T}^{-1} X(t, w)\right)=\left[I_{G} \cdot(L \bar{f})\right](X(t, w))
$$

for all $t$ and all $w \in \Omega$. Consequently, an elementary argument involving change of variables yields that

$$
f(X(t))-\int_{0}^{t}\left[I_{G} \cdot(\widetilde{L} f)\right](X(s)) d s
$$

is a $\widetilde{P}_{z}$-submartingale. This completes the proof.

Remark. Let Conditions II hold; in addition, let $\gamma_{j} \in C_{b}^{2}(\partial G)$. Let $T$ be defined as in (6). Since $\phi(0)=0$, the calculations leading to (12) show that for $2 \leqslant i, j \leqslant d, \partial^{2} g /\left(\partial y_{i} \partial y_{j}\right)=\partial^{2} g /\left(\partial z_{i} \partial z_{j}\right)$ on $\partial G$. Consequently, analogues of Lemma 2 and Theorem 1 hold in this case with $J$ given by (2) (in the $y$-variables $)$ and $\tilde{J}=\partial / \partial z_{1}+\frac{1}{2} \sum_{i, j=2}^{d} \alpha_{i j}(\cdot) \partial^{2} /\left(\partial z_{i} \partial z_{j}\right)$.

Hereafter, $L$ and $J$ will be as in (1) and (2), that is, in the $x$-variables. We need a few lemmas.

Lemma 3. Let Conditions I or II hold; let $\left\{P_{x}: x \in \bar{G}\right\}$ be the diffusion corresponding to $(L, J)$. Let $U$ be a bounded open set in $\bar{G}$. Then $\sup _{x \in U} E_{x}\left(\eta_{U}\right)<\infty$ and $\sup _{x \in U} E_{x}\left(\xi\left(\eta_{U}\right)\right)<\infty$, where $\xi$ is as in (3) and $\eta_{U}=\inf \{t \geqslant 0: X(t) \notin U\}$.

Proof. Let $h \in C_{b}^{2}(\bar{G})$ be such that $h(x)=e^{q x_{1}}$ for $x=\left(x_{1}, \ldots, x_{d}\right)$ in $\bar{U}$ and $q$ is a suitable positive constant so that $L h \geqslant 1$ in $\bar{U}$. Note that $J h \geqslant q>0$ on $\partial G \cap U$. By (3b) and optional sampling theorem, for every $T>0$

$$
\begin{gathered}
E_{x}\left[h\left(X\left(\eta_{U} \wedge T\right)\right)-h(X(0))-\int_{0}^{\eta_{U} \wedge T}\left[I_{G} \cdot(L h)\right](X(u)) d u\right. \\
\left.-\int_{0}^{\eta_{U} \wedge T} J h(X(u)) d \xi(u)\right]=0
\end{gathered}
$$

for $x \in U$ and $X(0)=x$. Since $h$ is bounded, $L h \geqslant 1$ in $\bar{U}$ and $J h>0$ on $\partial G$; by (16) and monotone convergence theorem it follows that $\sup _{x \in U} E_{x}\left(\eta_{U}\right)<\infty$. Again, since $h$ is bounded, $J h \geqslant q>0$ on $\partial G \cap U$ and 
$L h>0$ in $\bar{U}$, by (16) and monotone convergence theorem it follows that $\sup _{x \in U} E_{x}\left(\xi\left(\eta_{U}\right)\right)<\infty$.

Fix $\zeta \in \bar{G}$. For $x \in \bar{G}$ such that $x \neq \zeta$, define

$$
\begin{aligned}
A_{\zeta}(x) & =\sum_{i, j=1}^{d} a_{i j}(x) \frac{\left(x_{i}-\zeta_{i}\right)\left(x_{i}-\zeta_{j}\right)}{|x-\zeta|^{2}}, \\
B(x) & =\sum_{i=1}^{d} a_{i i}(x), C_{\zeta}(x)=2 \sum_{i=1}^{d} b_{i}(x)\left(x_{i}-\zeta_{i}\right) .
\end{aligned}
$$

For $r>0$, define

$$
\begin{aligned}
& \bar{\beta}_{\zeta}(r)=\sup _{|x-\zeta|=r} \frac{B(x)-A_{\zeta}(x)+C_{\zeta}(x)}{A_{\zeta}(x)}, \\
& \boldsymbol{\beta}_{\zeta}(r)=\inf _{|x-\zeta|=r} \frac{B(x)-A_{\zeta}(x)+C_{\zeta}(x)}{A_{\zeta}(x)} .
\end{aligned}
$$

Let $c>0$. Define for $r \geqslant c$,

$$
\begin{array}{ll}
\bar{I}_{c, \zeta}(r)=\int_{c}^{r} \frac{1}{u} \bar{\beta}_{\zeta}(u) d u, & \mathbf{I}_{c, \zeta}(r)=\int_{c}^{r} \frac{1}{u} \boldsymbol{\beta}_{\zeta \zeta}(u) d u, \\
\bar{F}_{c, \zeta}(r)=\int_{c}^{r} \exp \left(-\bar{I}_{c, \zeta}(u)\right) d u, & \mathbf{F}_{c, \zeta}(r)=\int_{c}^{r} \exp \left(-\mathbf{I}_{c, \zeta}(u)\right) d u,
\end{array}
$$

and let $\bar{f}_{c, \zeta}(x)=\bar{F}_{c, \zeta}(|x-\zeta|)$ and $\mathbf{f}_{c, \zeta}(x)=\mathbf{F}_{c, \zeta}(|x-\zeta|)$.

Let $H$ be a real-valued twice continuously differentiable function on $(0, \infty)$, and let $h(x)=H(|x-\zeta|)$. Then it is easily seen that for $|x-\zeta|>0$,

$$
2 L h(x)=A_{\zeta}(x) H^{\prime \prime}(|x-\zeta|)+\frac{H^{\prime}(|x-\zeta|)}{|x-\zeta|}\left(B(x)-A_{\zeta}(x)+C_{\zeta}(x)\right) .
$$

LeMma 4. Let Conditions I or II hold; let $\zeta \in \bar{G}$ be fixed. Let $c$ and $n$ be fixed real numbers such that $c<n$; let $x \in \bar{G}$ be such that $c<|x-\zeta|<n$; and let $\tau_{n}=\inf \{t \geqslant 0:|X(t)-\zeta|=c$ or $n\}$. Then

$$
\begin{aligned}
& \frac{\mathbf{F}_{c, \zeta}(|x-\zeta|)}{\mathbf{F}_{c, \zeta}(n)}+\frac{1}{\mathbf{F}_{c, \zeta}(n)} E_{x}\left[\int_{0}^{\tau_{n}} J \mathbf{f}_{c, \zeta}(X(u)) d \xi(u)\right] \\
& \quad \leqslant P_{x}\left(\tau_{\partial B(\zeta \cdot n)}<\tau_{\partial B(\zeta \cdot c)}\right) \\
& \quad \leqslant \frac{\bar{F}_{c, \zeta}(|x-\zeta|)}{\bar{F}_{c, \zeta}(n)}+\frac{1}{\bar{F}_{c, \zeta}(n)} E_{x}\left[\int_{0}^{\tau_{n}} J \bar{f}_{c, \zeta}(X(u)) d \xi(u)\right],
\end{aligned}
$$

where for a closed set $K$ in $\bar{G}, \tau_{K}=\inf \{t \geqslant 0: X(t) \in K\}$. 
Proof. Note that, by Lemma $3, \tau_{n}<\infty$ a.s. $P_{x}$. We apply (3b) to the functions $\mathbf{f}_{c, \zeta}$ and $\bar{f}_{c, \zeta}$ and proceed as in the proof of Lemma 2.1 in Bhattacharya and Ramasubramanian [2]; finally an application of the optional sampling theorem yields the lemma. We omit the details.

Remark. Suppose $L$ transforms smooth radial functions into smooth radial functions. Further, let $J=\partial / \partial x_{1}$. Also, let $\zeta=0$ for simplicity. Then $A_{0}(x)$ and $B(x)+C_{0}(x)$ are easily seen to be radial functions; consequently $\bar{\beta} \equiv \boldsymbol{\beta}$. Also $J \bar{f} \equiv J \mathbf{f} \equiv 0$ on $\partial G$. Hence (18) becomes

$$
\frac{\mathbf{F}_{c, 0}(|x|)}{\mathbf{F}_{c, 0}(n)}=P_{x}\left(\tau_{\partial B(0: n)}<\tau_{\partial B(0: c)}\right)=\frac{\bar{F}_{c, 0}(|x|)}{\bar{F}_{c, 0}(n)} .
$$

Since $L$ transforms radial functions into radial functions, by (17), it can be seen that solving $L h(x)=0$ in $c<|x|<n$ is reduced to solving a (1-dimensional) second-order ordinary differential equation in the interval $(c, n)$. The latter can be done easily, and (19) thus gives the solution to the problem:

$$
\begin{array}{rlll}
L h(x)=0 & \text { for } c<|x|<n, & J h(x)=0 & \text { for } x \in \partial G, \\
h(x)=1 & \text { for }|x|=n, & h(x)=0 & \text { for }|x|=c .
\end{array}
$$

In the general case, for $\zeta \in \partial G, P_{x}\left(\tau_{\partial B(\zeta: n)}<\tau_{\partial B(\zeta: c)}\right)$ is bounded above and below by similar radial functions (which are harmonic for an elliptic operator which transforms radial functions into radial functions), plus correction terms depending essentially on the boundary conditions (cf. see $[1,2]$.

We are now in a position to prove our main theorem.

THEOREM 2. Let Conditions I or II hold, and let $\zeta \in \partial G$. Then for any $n>0$ and any $x$ such that $0<|x-\zeta| \leqslant n$,

$$
\lim _{c \downarrow 0} P_{x}\left(\tau_{\partial B(\zeta ; n)}<\tau_{\partial B(\zeta ; c)}\right)=1 .
$$

Consequently, the diffusion does not hit a point on the boundary specified in advance.

Proof. (i) Let Conditions I hold. In view of Theorem 1 it is sufficient to consider the case $J=\partial / \partial x_{1}$. In such a case note that $J \mathbf{f}_{c, \zeta} \equiv 0$ on $\partial G$. Then, as $\mathbf{F}_{c, \zeta}(|x-\zeta|) / \mathbf{F}_{c, \zeta}(n) \rightarrow 1$ as $c \downarrow 0$ for any $n>0$ and any $0<|x-\zeta| \leqslant n$, (20) follows from (18). 
(ii) Let Conditions II hold. Let $x \in \bar{G}$ be fixed and $x \neq \zeta$. Let $n>0$ be fixed. Let $\varepsilon>0$ be given. Choose $c>0$ such that

$$
\frac{\mathbf{F}_{c, \zeta}(|x-\zeta|)}{\mathbf{F}_{c, \zeta}(n)}>(1-\varepsilon)
$$

Note that constants $\Gamma_{j}, j=2, \ldots, d$ can be chosen so that

$$
\hat{J} \mathbf{f}_{c, \zeta}(y) \geqslant 0, \quad \text { for } c \leqslant|y-\zeta| \leqslant n, y \in \partial G
$$

where

$$
\hat{J}=J+\sum_{i, j=2}^{d} \tilde{\sigma}_{i j}(\cdot) \Gamma_{j} \frac{\partial}{\partial x_{i}} .
$$

Let $Q_{x}^{c}$ be the diffusion corresponding to $(L, \hat{J})$, starting at $x$. Note that, by a Girsanov-type theorem (Nakao and Shiga [6, pp. 453, 468]),

$$
\left.\psi_{c, t} \equiv \frac{d Q_{x}^{c}}{d P_{x}}\right|_{j \theta_{i}}=\exp \left\{\sum_{j=2}^{d} \Gamma_{j} B_{j}(\xi(t))-\frac{1}{2} \sum_{j=2}^{d} \Gamma_{j}^{2} \xi(t)\right\},
$$

where $\left(B_{2}(s), \ldots, B_{d}(s)\right)$ is a $(d-1)$-dimensional $P_{x}$-Brownian motion independent of $\xi(t)$.

Write $A=\left\{\tau_{\partial B(\zeta: n)}<\tau_{\partial B(\zeta: c)}\right\}$ and $A_{t}=\left\{\left(\tau_{\tau_{B B(\zeta: n)}} \wedge t\right)<\left(\tau_{\partial B(\zeta: c)} \wedge t\right)\right\}$. By (18), (21), (22) applied to the $(L, \hat{J})$-diffusion, we get

$$
Q_{x}^{c}(A)>(1-\varepsilon) .
$$

Consequently, $Q_{x}^{c}\left(A_{t}\right)>(1-\varepsilon)$; and hence

$$
\int_{A_{t}} \psi_{c, l} d P_{x}>(1-\varepsilon)
$$

Note that $P_{x}\left(\psi_{c, t}>1\right) \rightarrow 0$ as $t \rightarrow \infty$. Hence (23) implies that $\lim _{t \rightarrow \infty} P_{x}\left(A_{t}\right)>(1-\varepsilon)$. Thus $P_{x}(A)>(1-\varepsilon)$, whence $(20)$ follows. This completes the proof.

We now give two applications.

Corollary 1. Let Conditions I or II hold. Let $D$ be a bounded open set in $\bar{G}$ satisfying an exterior cone condition (in $\bar{G}$ ) and such that $\partial D \cap \partial G$ is a finite set. Let $\tau=\inf \{t \geqslant 0: X(t) \notin D\}$. Then $\tau$ is continuous $P_{x}$-a.s. for any $x \in D$.

Proof. Set $\tau^{\prime}=\inf \{t \geqslant 0: X(t) \notin \bar{D}\}$. It can be seen that $\tau$ is upper semicontinuous and that $\tau^{\prime}$ is lower semicontinuous. Therefore, it is suf- 
ficient to prove that $P_{x}\left(\tau=\tau^{\prime}\right)=1$. Since, by Theorem $2, P_{x}(X(\tau) \in \partial G)=0$, it is sufficient to prove that $P_{y}\left(\tau^{\prime}>0\right)=0$ for any $y \in \partial D, y \notin \partial G$. Because of the $0-1$ law, it is sufficient to prove that $P_{y}\left(\tau^{\prime}>0\right) \neq 1$ for any $y \in \partial D$, $y \notin \partial G$. This now follows from the exterior cone condition and the support theorem of Stroock and Varadhan [8, Ex.6.7.5]).

Corollary 2. Let Conditions I or II hold; let $\rho \equiv 0$. Let $D$ and $\tau$ be as in the preceding lemma. Let $f, g, h$ be bounded and continuous functions respectively on $D, \partial D, \partial G$. Then

$$
u(x)=E_{x}\left[g(X(\tau))-\int_{0}^{\tau} f(X(s)) d s-\int_{0}^{\tau} h(X(s)) d \xi(s)\right]
$$

is continuous on $D$.

Proof. In view of Lemma 3, note that $u$ is well defined and bounded. By the preceding corollary and Feller continuity, the corollary follows.

Remark. Note that $u$ defined as in the preceding corollary is the unique solution to the boundary value problem

$$
L u=f \quad \text { on } D, \quad u=g \quad \text { on } \partial D, \quad J u=h \quad \text { on } \partial G ;
$$

that is,

$$
u(X(t \wedge \tau))-\int_{0}^{t \wedge \tau} f(X(s)) d s-\int_{0}^{t \wedge \tau} h(X(s)) d \xi(s)
$$

is a $P_{x}$-martingale, and $u=g$ on $\partial D$. If $D$ is as before and is connected, $f \geqslant 0$ in $D$ and if $u(x)=0$ for some $x \in D, x \notin \partial G$, then by the preceding corollary and Lemma 2.3 of Bhattacharya [1] it follows that $u \equiv 0$ in $\bar{D}$.

\section{ACKNOWLEDGMENTS}

The author wishes to thank Dr. P. Jothilingam for a useful discussion. Thanks are also due to a referee for valuable suggestions for improvement.

\section{REFERENCES}

[1] Bhattacharya, R. N. (1978). Criteria for recurrence and existence of invariant measures for multidimensional diffusions, Ann. Probab. 6, 541-553.

[2] Bhattacharya, R. N. and Ramasubramanian, S. (1982). Recurrence and ergodicity of diffusions. J. Multivariate Anat. 12, 95-122. 
[3] El Karoui, N. (1975). Processes de reflexion dans $R^{n}$. Seminaire de Probabilities IX. Universite de Strasbourg, pp. 534-554. Lecture Notes in Math., Vol.465. Springer-Verlag, New York/Berlin.

[4] McKean, H. P. (1969). Stochastic Integrals. Academic Press, New York.

[5] NAKAO, S. (1972). On the existence of solutions of stochastic differential equations with boundary conditions, J. Math. Kyoto Univ. 12, 151-178.

[6] NaKaO, S. AND SHIGA, T. (1972). On the uniqueness of solutions of stochastic differential equations with boundary conditions. J. Math. Kyoto Univ. 12, 451-478.

[7] Stroock, D. W. And Varadhan, S. R. S. (1971). Diffusion processes with boundary conditions. Comm. Pure Appl. Math. 24, 147-225.

[8] Stroock, D. W. And Varadhan, S. R. S. (1979). Multidimentional Diffusion Processes. Springer-Verlag, New York.

[9] Watanabe. S. (1971). On stochastic differential equations with multidimensional diffusion processes with boundary conditions-I, II. J. Math. Kyoto Univ. 11, 169-180, $545-551$. 